C2008 IEEE. Personal use of this material is permitted. However, permission to reprint/republish this material for advertising or promotional purposes or for creating new collective works for resale or redistribution to servers or lists, or to reuse any copyrighted component of this work in other works must be obtained from the IEEE. 


\section{Performance Analysis of Signal-to-Noise Ratio Estimators in AWGN and Fading Channels}

\author{
Lenin Gopal \\ Department of Electrical/Communication Engineering and \\ Computing, School of Engineering and Science, Curtin \\ University of Technology, Miri, Malaysia \\ lenin@curtin.edu.my
}

\begin{abstract}
Additive White Gaussian Noise (AWGN) and Rayleigh fading severely degrade the performance of the wireless communication systems. Most of the wireless communication systems require knowledge of the channel Signal-to-Noise ratio. In this paper a few methods are proposed to estimate the SNR in the presence of AWGN and Rayleigh fading. The mean square error (MSE) and root mean square error (RMSE) are used as performance measures. Simulation result shows that the newly proposed estimators $\mathrm{ml}_{\text {fad }}$ can provide better performance in most circumstances under AWGN and Rayleigh fading channels.
\end{abstract}

Keywords: Estimation, Signal-to-Noise Ratio (SNR), AWGN noise, Rayleigh fading.

\section{INTRODUCTION}

Additive white Gaussian noise and multipath fading severely degrade the performance of wireless communication systems. In various communication systems, the signal-to-noise ratio (SNR) is one of the parameters that give the quality measure of their communication links. Many wireless communication systems require knowledge of the channel signal-to-noise ratio. In the CDMA cellular system, SNR is a crucial input parameter for satisfactory close-loop power control performance [1]. Various SNR estimation techniques for AWGN [1] to [8] and fading channel have been published in the literature over the last few decades. Most of the literatures derived the SNR estimates separately for AWGN channels [1] to [8] and fading channels. Recently, [11] derived an SNR estimate based on a statistical ratio of observables over a block of data when the channel undergoes Nakagami fading. The derived SNR estimator is used for Rayleigh fading (when the Nakagami parameter $m \rightarrow 1$ ) and AWGN channel (when the Nakagami parameter $m \rightarrow \infty$ ). However, this study does not include the case of AWGN and Rayleigh fading. In this paper, we study the SNR estimation in the presence of simultaneous AWGN and fading processes. The SNR estimation techniques for AWGN channel presented in [1] cannot be used directly without modification in a fading channel. Similarly, local mean power estimation techniques that work in Rayleigh fading channel [9] cannot be used directly in an AWGN channel.

In this paper, three SNR estimation techniques, namely Sample average, $\mathrm{ml}_{\mathrm{awgn}}$ and $\mathrm{ml}_{\text {fad }}$ SNR estimators, are proposed to estimate the SNR in the presence of AWGN and Rayleigh fading. First part of the paper introduces the system model for SNR estimation. Next, the details of the three SNR estimators are proposed and presented. The performance of the

\author{
Moh Lim Sim \\ Faculty of Engineering, Multimedia University, \\ Cyberjaya, Kuala Lumpur, Malaysia \\ mlsim@mmu.edu.my
}

three SNR estimators and conclusion are included in the subsequent sections.

\section{PROPOSED SYSTEM MODEL}

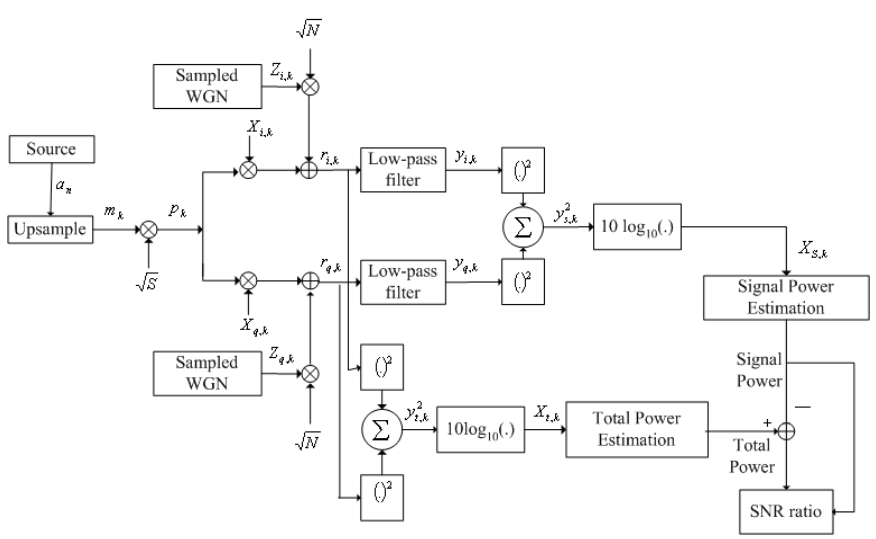

Figure 1. System model for SNR estimation in the presence of AWGN and fading channel

The system model for the performance analysis of SNR estimators in the presence of AWGN and Rayleigh fading is shown in Figure 1. A block of $N_{S Y M}$ binary sources symbols are generated and it is represented by $a_{n}$. The generated binary source symbol is up-sampled. The up-sampled binary message sequence is represented by $m_{k}$. The up-sampled binary sequence is corrupted by fading and AWGN. The in-phase and quadrature-phase components of the signal presented to the receiver are

$$
\begin{aligned}
& r_{i, k}=m_{k} \cdot \sqrt{S} \cdot X_{i, k}+\sqrt{N} \cdot Z_{i, k} \\
& r_{q, k}=m_{k} \cdot \sqrt{S} \cdot X_{q, k}+\sqrt{N} \cdot Z_{q, k}
\end{aligned}
$$

$Z_{i, k}$ and $Z_{q, k}$ are the sampled, zero mean in-phase and quadrature-phase of AWGN of unit variance, $S$ is the signal power scale factor, $N$ is the noise power scale factor, and $X_{i, k}, X_{q, k}$ are the in-phase and quadrature-phase components of the Rayleigh fading coefficient. Low-pass filter is used to 
filter out the noise from the received signal. The samples of the received signal after the low-pass filter can be expressed as

$$
\begin{gathered}
y_{i, k}=r_{i, k} \otimes h_{l} \\
y_{q, k}=r_{q, k} \otimes h_{l}
\end{gathered}
$$

where $\otimes$ denotes discrete convolution, and $h_{l}$ represents the low-pass filter coefficient (with rolloff, $\alpha=0.5$ and $L=128$ tap coefficients).

\section{SNR ESTIMATION}

In this section, new techniques are proposed to estimate the SNR for Rayleigh fading channels and signals corrupted by AWGN. In these techniques, the signal and noise powers are estimated separately. The outputs of the low-pass filter can be squared and summed as

$$
y_{s, k}^{2}=y_{i, k}^{2}+y_{q, k}^{2}
$$

where $y_{s, k}$ is Rayleigh distributed, $y_{i, k}$ and $y_{q, k}$ are in-phase and quadrature-phase components of the $k^{\text {th }}$-sample received signal at the output of the low-pass filter. The filtered output signal $y_{s, k}$ is fed to a log amplifier before to the signal power estimator. The samples are in decibels because the output of the amplifiers often has a logarithmic characteristic. Then, the output of the log amplifier can be expressed as

$$
X_{s, k}=20 \log y_{s, k}
$$

The distribution function of the output signal is called the Antilog Rayleigh (ALR). The local mean power estimators are used to estimate the received signal power at this stage. The total received power is estimated from the unfiltered received samples:

$$
y_{t, k}^{2}=r_{i, k}^{2}+r_{q, k}^{2}
$$

where $r_{i, k}$ and $r_{q, k}$ are the in-phase and quadrature-phase component of the $k^{\text {th }}$-sample received signal at the input of the low-pass filter. Then, the output of the log amplifier can be expressed as

$$
X_{t, k}=20 \log y_{t, k}
$$

The estimated signal power is subtracted from the estimated total power to obtain the noise power. The local mean power estimator[4], Sample average (denoted as sample), can be modified to estimate the SNR in the presence of AWGN and Rayleigh fading channels as shown in Figure 1. Two new SNR estimators are proposed namely $\mathrm{ml}_{\text {awgn }}$ and $\mathrm{ml}_{\text {fad }}$ SNR estimators. The two estimators $\left(\mathrm{ml}_{\text {awgn }}\right.$ and $\left.\mathrm{ml}_{\mathrm{fad}}\right)$ are modified from the Maximum Likelihood SNR estimator for AWGN channel [1].

The SNR estimation process is divided into 2 phases. In phase 1, the short-term SNR will be estimated where the noise effect would be averaged out. Small number of samples $N_{S}$ would be used to ensure that the samples used is highly correlated. Meanwhile phase 2 is required to provide averaging of SNR of the received signal over longer duration of time in order a more accurate of long term time-average of the SNR can be obtained. SNR estimates from phase 1 may be at various strength of the fading channel. In order to give an average over the varying strength of the fading channel, $N_{S}$ number of samples of short term SNR estimates from phase 1 would be used to provide a long term estimate.

Phase 1: Short-term SNR is estimated as follows:

a) Sample Average Estimator

$$
\hat{\rho}_{\text {sample }} \text { Est }, j=\frac{\sum_{k N_{S}+1}^{N_{S}(j+1)} 10^{X_{s, k} / 10}}{\sum_{k=j N_{S}+1}^{N_{S}(j+1)} 10^{X_{t, k} / 10}-\sum_{k=\sum_{S}+1}^{N_{S}(j+1)} 10^{X_{s, k} / 10}}
$$

b) $\underline{\mathrm{ml}}_{\mathrm{awgn}}$ Estimator

$$
\hat{\rho}_{m_{\text {luwg } n} \text { Est } j}=\frac{\frac{1}{P_{j}^{2}}\left[\frac{1}{N_{S}} \sum_{k=j N_{S}+1}^{N_{S}(j+1)} r_{i, k} m_{k}^{*}\right]^{2}+\frac{1}{P_{j}^{2}}\left[\frac{1}{N_{S}} \sum_{k=j N_{S}+1}^{N_{S}(j+1)} r_{q, k} m_{k}^{*}\right]^{2}}{\frac{1}{N_{S}} \sum_{k=j N_{S}+1}^{N_{S}(j+1)} 10^{X_{t, k} / 10}-\left[\frac{1}{P_{j}^{2}}\left[\frac{1}{N_{S}} \sum_{k=j N_{S}+1}^{N_{S}(j+1)} r_{i, k} m_{k}^{*}\right]^{2}+\frac{1}{P_{j}^{2}}\left[\frac{1}{N_{S}} \sum_{k=j N_{S}+1}^{N_{S}(j+1)} r_{q, k} m_{k}^{*}\right]^{2}\right]}
$$

c) $\underline{\mathrm{ml}}_{\text {fad }}$ Estimator

$\hat{\rho}_{m l_{\text {fad }} E s t, j}=\frac{\frac{1}{P_{j}^{2}}\left[\frac{1}{N_{S}} \sum_{k=j N_{S}+1}^{N_{S}(j+1)} r_{i, k} m_{n, k}^{*}\right]^{2}+\frac{1}{P_{j}^{2}}\left[\frac{1}{N_{S}} \sum_{k=j N_{S}+1}^{N_{S}(j+1)} r_{q, k} m_{n, k}^{*}\right]^{2}}{\frac{1}{N_{S}} \sum_{k=j N_{S}+1}^{N_{S}(j+1)} 10^{X_{t, k} / 10}-\frac{1}{N_{S}} \sum_{k=j N_{S}+1}^{N_{S}(j+1)} 10^{X_{S, k} / 10}}$

where $P_{j}=\frac{1}{N_{S}} \sum_{k=j N_{S}+1}^{N_{S}(j+1)}\left(m_{k}^{*}\right)^{2}$, and $m_{k}^{*}$ denotes the data sequence formed at the receiver from a replica of the transmitted data and $N_{S}$ is the number of samples used in the short term SNR estimation. 
Phase 2: Long term SNR estimation

a) Sample average estimator

$$
\hat{\rho}_{\text {sample }, i}=\frac{1}{N_{E}} \sum_{j=1+i N_{E}}^{N_{E}(i+1)} \hat{\rho}_{\text {sample }} E s t, j
$$

b) $\mathrm{ml}_{\text {awgn }} \underline{\text { estimator }}$

$$
\hat{\rho}_{m l_{\text {awg }}, i}=\frac{1}{N_{E}} \sum_{j=I+i N_{E}}^{N_{E}(i+1)} \hat{\rho}_{m l_{a w g n}-E s t, j}
$$

c) $\mathrm{ml}_{\text {fad }}$ estimator

$$
\hat{\rho}_{m l_{f a d}, i}=\frac{1}{N_{E}} \sum_{j=1+i N_{E}}^{N_{E}(i+1)} \hat{\rho}_{m l_{f a d}-E s t, j}
$$

where $N_{E}$ is the number of samples used in the long term SNR estimation.

\section{PERFORMANCE MEASURE}

In searching for optimal estimators, some performance measure criterion is needed. A common one is the Root Mean Square Error (RMSE), defined as

$$
\operatorname{RMSE}(\hat{\rho})=\sqrt{\frac{1}{N_{t}} \sum_{i=1}^{N_{t}} \Delta_{i}^{2}}
$$

where $\rho$ is the true SNR, subscript $i$ is the index which denotes the simulation run, $\Delta_{i}=\rho_{i}[d B]-\rho[d B]$, and $N_{t}$ is the total number of simulation run used to evaluate the performance of the estimator. The sample normalised Root Mean Square Error is computed accordingly to

$$
\operatorname{NRMSE}(\hat{\rho})=\operatorname{RMSE}(\hat{\rho}) / \rho
$$

The performance of the three estimators is also compared by using the Mean Error of the estimator. A good SNR estimator is the one that is unbiased or exhibits small Mean Error. The Mean Error of the estimator can be measured by

$$
\operatorname{ME}(\hat{\rho})=\frac{1}{N_{t}} \sum_{i=1}^{N_{t}} \Delta_{i}
$$

The sample normalised Mean Error is measured for each estimator by simulation from a number of estimates and it can be expressed as

$$
\operatorname{NME}(\hat{\rho})=\operatorname{ME}(\hat{\rho}) / \rho
$$

\section{SIMULATION RESULTS AND COMPARISONS}

In this section, the three SNR estimators are compared graphically by plotting the normalised Root Mean Square Error, and the normalised Mean Error of each estimator for the signal operating in the channel model described in Figure 1.

Simulations are performed for several fading frequency and SNR estimator errors are calculated. The values of $N_{E}$ and $N_{S}$ parameters are varied to study the performance of the SNR estimators. Number of samples generated per simulation run is 8192 samples.

\section{A. Effect of Doppler spread}

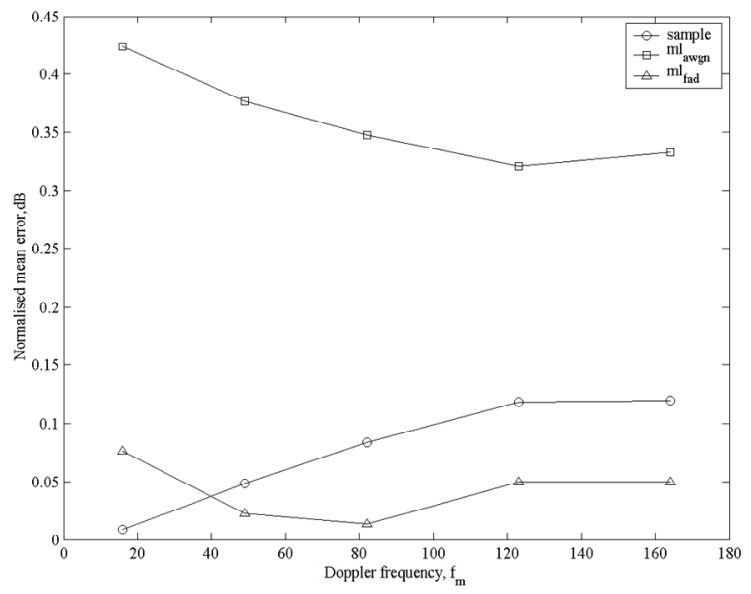

Figure 2. Normalised mean error, $\mathrm{dB}\left(f_{c}=200 \mathrm{~Hz}, N_{S}=4, N_{E}=110, S N R=5 \mathrm{~dB}\right)$

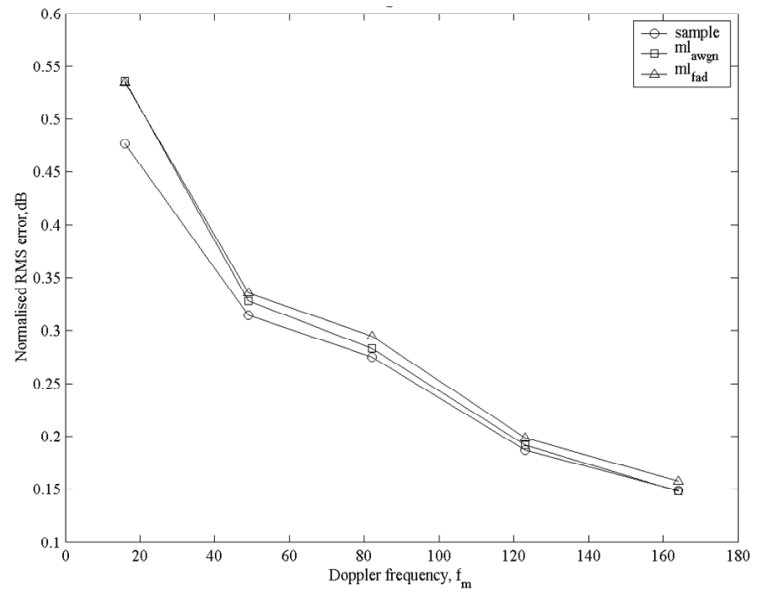

Figure 3. Normalised RMS error, $\mathrm{dB}\left(f_{c}=200 \mathrm{~Hz}, N_{S}=4, N_{E}=110, S N R=5 \mathrm{~dB}\right)$

Figure 2 and 3 show the normalised mean error and RMS error of the three estimators in the presence of the AWGN and Rayleigh fading channels. Mean error of the $\mathrm{ml}_{\text {awgn }}$ estimator decreases for increasing value of the Doppler frequency except at very high Doppler frequency. The $\mathrm{ml}_{\mathrm{awgn}}$ estimator has poor performance and it has very high mean error as compared to the other two estimators. Mean error of the Sample average 
estimator increases for increasing value of the Doppler frequency. The Sample average estimator has better performance as compared to the $\mathrm{ml}_{\mathrm{awgn}}$ estimator. Mean error of the $\mathrm{ml}_{\mathrm{fad}}$ estimator decreases for increasing value of the Doppler frequency. The $\mathrm{ml}_{\text {fad }}$ estimator has the best performance as compared to the other two estimators for Doppler frequencies $f_{m}>40 \mathrm{~Hz}$. Figure 3 shows that the three estimators have similar performance in terms of normalised RMS error. In addition, the normalised RMS error decreases at the Doppler frequency $f_{m}$ increases.

\section{B. Effect of SNR}

As SNR increases, the normalised mean error for Sample average and $\mathrm{ml}_{\text {awgn }}$ estimators first drop then increases as shown in figure 4 . The Sample average and $\mathrm{ml}_{\text {fad }}$ estimators have better performance as compared to the $\mathrm{ml}_{\mathrm{awgn}}$ estimator. The $\mathrm{ml}_{\mathrm{fa}}$ estimator performs very well at low SNR giving low mean error and performs comparable to the Sample average estimator at high SNR. Figure 5 shows that the normalised RMS errors of the three estimators are about the same order of magnitude and decrease for increasing value of SNR.

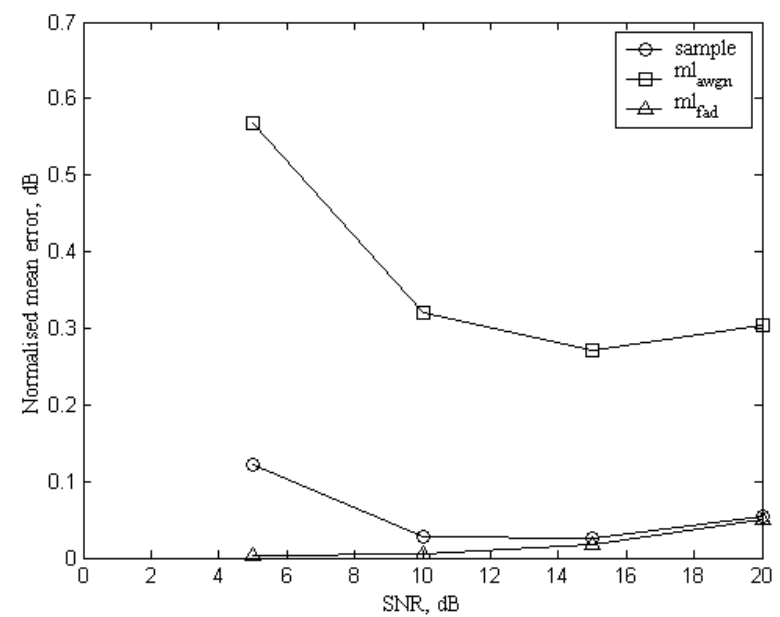

Figure 4. Normalised mean error, $\mathrm{dB}\left(f_{m}=49 \mathrm{~Hz}, f_{c}=200 \mathrm{~Hz}, N_{S}=4, N_{E}=230\right)$

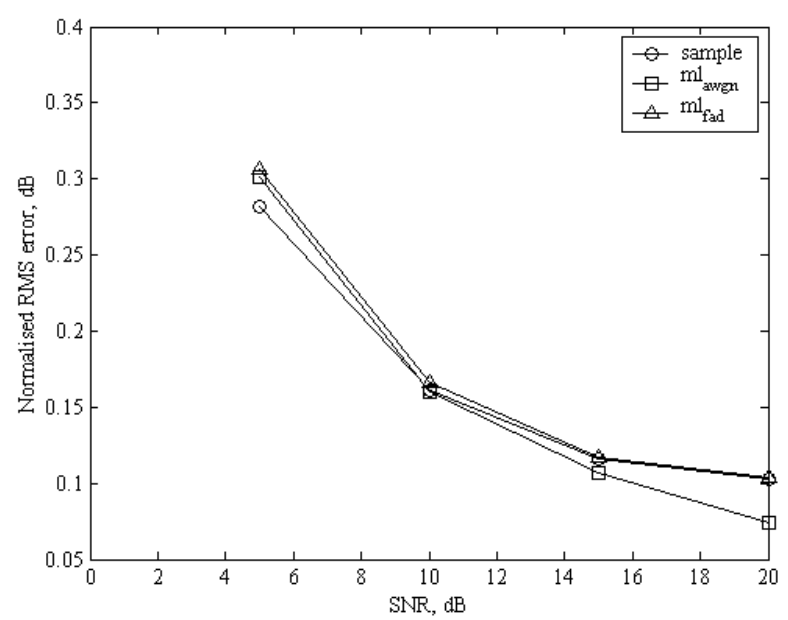

Figure 5. Normalised RMS error, dB $\left(f_{m}=49 \mathrm{~Hz}, N_{S}=4, f_{c}=200 \mathrm{~Hz}, N_{E}=230\right)$
C. Effect of $N_{S}$

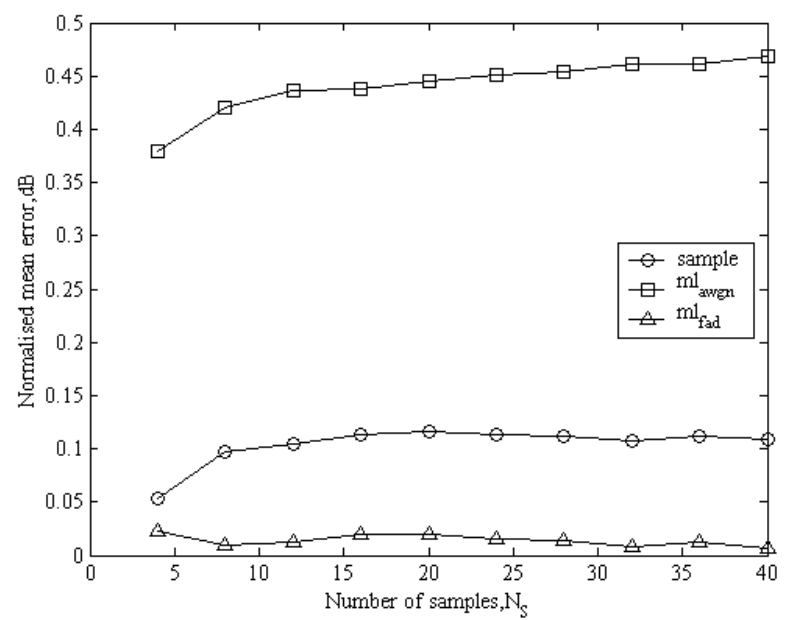

Figure 6. Normalised mean error, dB $\left(f_{m}=49 \mathrm{~Hz}, f_{c}=200 \mathrm{~Hz}, N_{E}=110, S N R=5\right.$ $\mathrm{dB})$

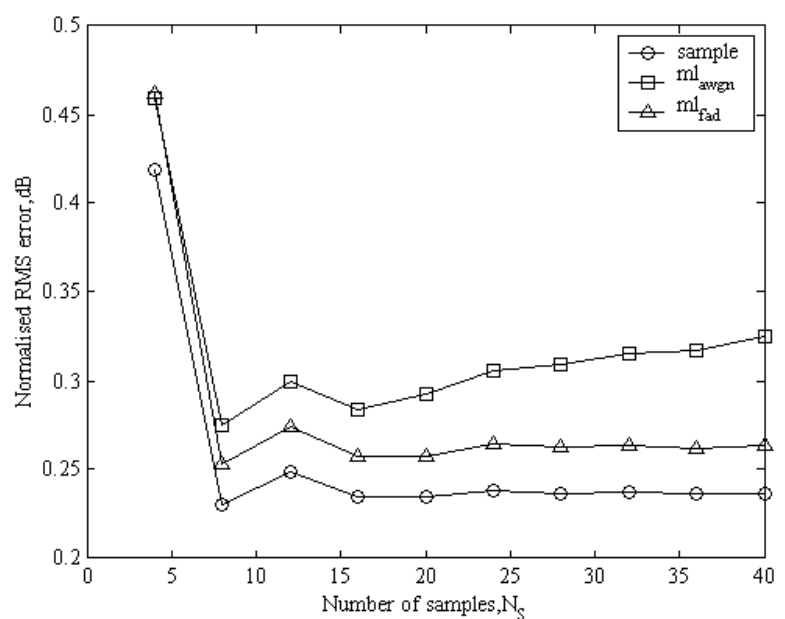

Figure 7. Normalised RMS error, $\mathrm{dB}\left(f_{m}=49 \mathrm{~Hz}, f_{c}=200 \mathrm{~Hz}, N_{E}=110, S N R=5\right.$ $\mathrm{dB})$

Normalised mean and RMS errors of the estimators is plotted in the Figures 6 and 7 with respect to $N_{S}$, the number of samples used for estimating short term SNR. In Figure 6, mean error of the $\mathrm{ml}_{\text {awgn }}$ and sample average estimators increase with $N_{S}$. At the low SNR of $5 \mathrm{~dB}$ and Doppler frequency of $49 \mathrm{~Hz}$, the performance of the $\mathrm{ml}_{\text {fad }}$ estimator is the best among the three estimators under study and has small mean error consistently over the entire tested range of $N_{S}$. From Figure 7 , the $\mathrm{ml}_{\text {awgn }}$ estimator has the worst RMS error performance, follows by the $\mathrm{ml}_{\text {fad }}$ and sample average estimators. The Normalised RMS error of the three estimators is minimum when the value of $N_{S}$ is 8 number of samples.

\section{Effect of $N_{E}$}

In Figure 8 and 9, number of samples used to determine the average of the estimated long term SNR, $N_{E}$ is varied to study the performance of the three SNR estimators. The mean error of the three estimators under study decrease for increasing 
value of $N_{E}$. In particular when $N_{E}$ increases beyond 30 , the estimators' mean error performance becomes saturated. The $\mathrm{ml}_{\text {awgn }}$ estimator has the worst performance as compared to the other two estimators which perform similarly. From Figure 9, the RMS errors of three estimators decrease for increasing $N_{E}$. In contrast to the mean error performance, the $\mathrm{ml}_{\text {awgn }}$ estimator has the best RMS error performance while the other two estimators perform similarly.

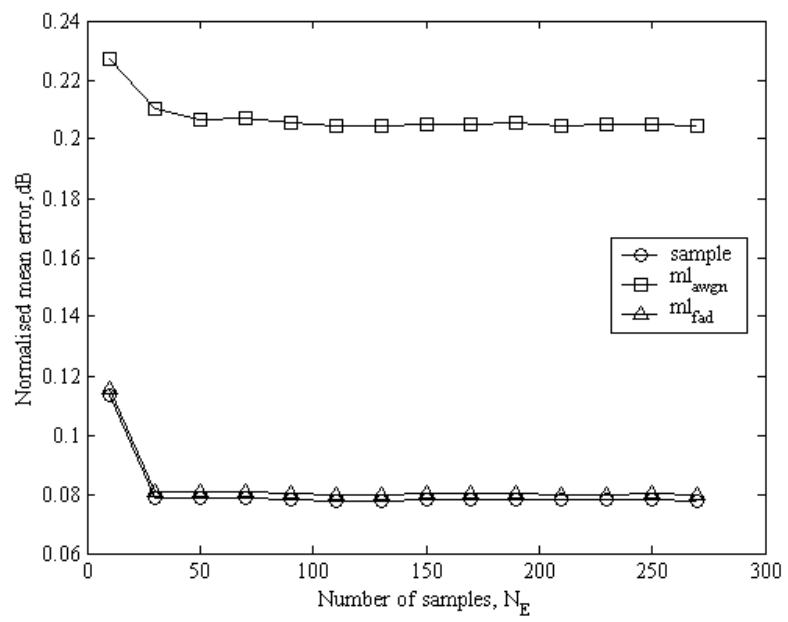

Figure 8. Normalised mean error, $\mathrm{dB}\left(f_{m}=16 \mathrm{~Hz}, f_{c}=200 \mathrm{~Hz}, N_{S}=4, S N R=5\right.$ $\mathrm{dB})$

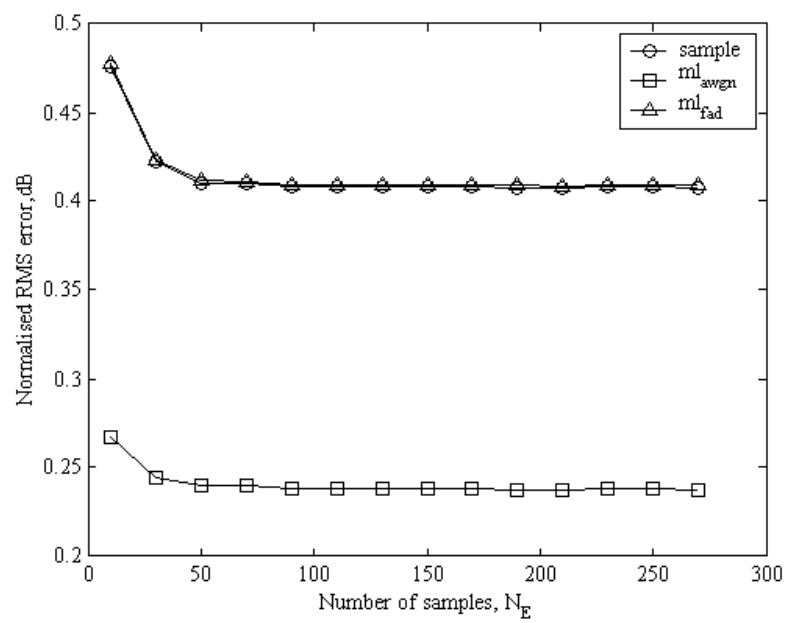

Figure 9. Normalised RMS error $\left(f_{m}=16 \mathrm{~Hz}, f_{c}=200 \mathrm{~Hz}, N_{S}=4, S N R=5 \mathrm{~dB}\right)$.

\section{CONCLUSiON}

Three new signal-to-noise ratio estimation methods for AWGN and fading channel are proposed and their performances are investigated. The simulation parameters which include the number of samples for short term SNR, $N_{S}$, number of samples for long term SNR estimation, $N_{E}$, Doppler frequency and the actual signal-to-noise ratio are varied to study the performance of the three estimators. Generally, the Sample average and $\mathrm{ml}_{\text {fad }}$ estimators perform relatively better as compared to the $\mathrm{ml}_{\text {awgn }}$ estimator. Overall, among the three estimators under studied, the $\mathrm{ml}_{\text {fad }}$ estimator is recommended for practical application as it gives the best performance at low SNR and $f_{m}>50 \mathrm{~Hz}$. Meanwhile, it performs similar as other estimators in terms of mean error at other conditions.

\section{REFERENCES}

[1] D. R. Pauluzzi and N. C. Beaulieu, "A comparison of SNR estimation techniques for the AWGN channel," IEEE Trans. Commun., vol.48, pp. 1681-1691, Oct 2000

[2] Bin Li, Robert DiFazio and Ariela Zeira, "A Low Bias Algorithm to Estimate Negative SNRs in an AWGN Channel", IEEE Commun Lett, 2002, vol.6, no 11, pp 469-471

[3] R.Matzner and F.Engleberger, "An SNR estimation algorithm using fourth order moments', proc.IEE Int.Symp.Information Theory, Trondheim, Norway, 1994, p.119.

[4] Rolf Matzner, "An SNR estimation algorithm for complex baseband signals using higher order statistics", Facta Universitatis, series: Electronics and Energetics, 1993, no. 6, pp. 41-52.

[5] R.M.Gagliardi and C.M. Thomas, "PCM data reliability monitoring through estimation of signal-to-noise ratio", IEEE Trans. Commun. Technol, 1968, vol. 16. no.2, pp.470-486.

[6] C. E. Gilchriest, "Signal-to-noise monitoring", Spacing Programs Summary 37-27, IV, Jet Propulsion Laboratory, Pasadena, 1967, CA, pp. 169-184.

[7] T.R. Benedict, T.T. Soong, "The joint estimation of signal and noise from the sum envelope", IEEE Trans. Inform. Theory, 1967, vol 13, no. 3, pp. 447-454.

[8] C.M.Thomas, "Maximum likelihood estimation of signal-to-noise ratio", Ph.D. dissertation, University of Southern California, Los Angeles, 1967.

[9] D. Wong and D. C. Cox, "Estimating Local Mean Signal Power Level in a Rayleigh Fading Environment", IEEE Transactions on Vehicular Technology, 1999, Vol-48, No-3, pp.956-959.

[10] Tao Jiang, N. D. Sidiropoulos, and G. B. Giannakis, "Kalman Filtering for Power Estimation in Mobile Communications", IEEE Trans. On Wireless Communications, 2003, Vol.2, No-1, pp.151-161.

[11] A. Ramesh, A. Chockalingam, and L. B. Milstein, "SNR Estimation in Generalized Fading Channels and its Application to Turbo Decoding", Proc. IEEE ICC'2001, 2001, pp. 1094-1098. 